\title{
Stability of multiquarks in a simple string model
}

\author{
J. Vijande, ${ }^{1,2, *}$ A. Valcarce,,$^{2, \dagger}$ and J.-M. Richard ${ }^{3, *}$ \\ ${ }^{1}$ Departamento de Física Teórica and IFIC, Universidad de Valencia - CSIC, 46100 Burjassot, Valencia, Spain \\ ${ }^{2}$ Departamento de Física Fundamental, Universidad de Salamanca, 37008 Salamanca, Spain \\ ${ }^{3}$ Laboratoire de Physique Subatomique et Cosmologie, Université Joseph Fourier-INPG-IN2P3-CNRS, \\ 53, Avenue des Martyrs, 38026 Grenoble, France \\ (Received 26 July 2007; published 17 December 2007)
}

\begin{abstract}
A simple string model inspired by the strong-coupling regime of quantum chromodynamics is used as a potential for studying the spectrum of multiquark systems with two quarks and two antiquarks, with a careful treatment of the four-body problem. It is found that the ground state is stable, lying below the threshold for dissociation into two isolated mesons.
\end{abstract}

DOI: 10.1103/PhysRevD.76.114013

PACS numbers: 12.39.Jh, 12.40.Yx

The question of the existence of multiquark systems is almost as old as the concept of quarks, see, e.g., [1], in particular, the paper by R. H. Dalitz therein. Since the early days of hadron spectroscopy within the quark approach, many studies have been devoted to multiquark states. Of particular interest are hadrons with exotic quantum numbers that cannot be matched by any quark-antiquark $(q \bar{q})$ or three-quark $(q q q)$ configuration, and among them, the states, if any, which are bound below the threshold for dissociation into two ordinary hadrons and thus are narrow and should show up clearly in the experimental spectrum.

The present contribution belongs to the class of constituent quark models: an explicit set of rules is adopted to mimic the interaction of quarks in quantum chromodynamics (QCD) and, within this framework, the 2-body, 3-body, and higher few-body problems are solved as accurately as possible to examine whether quarks tend to split into small $(q \bar{q})$ and $(q q q)$ clusters or sometimes find it energetically more favorable to form multiquark clusters. After a series of estimates within the bag model (see, e.g., [2]), there have been several attempts with potential models, using the powerful few-body techniques developed in atomic and nuclear physics.

Several dynamical ingredients have been identified along the years as possible sources of multiquark binding. The best known is probably chromomagnetism [3]: the spin-color operator $\boldsymbol{\sigma}_{i} \cdot \boldsymbol{\sigma}_{j} \tilde{\lambda}_{i} \cdot \tilde{\lambda}_{j}$, which is encountered in the spin-dependent part of one-gluon exchange gives rise to remarkable coherence effects, and gives in some multiquark clusters some attraction that is larger than in its decay products. This mechanism was proposed, in particular, for the H dibaryon (uuddss) [4], tentatively below the $\Lambda \Lambda$ threshold, and for the 1987 version of the heavy pentaquark $(Q \bar{q} \bar{q} \bar{q} \bar{q})$ [5]. The chromomagnetic scenario has, however, difficulties: the first optimistic predictions carried out in the limit of exact flavor SU(3) symmetry, and

\footnotetext{
*javier.vijande@uv.es

valcarce@usal.es

jean-marc.richard@1psc.in2p3.fr
}

using short-range correlation coefficients borrowed from ordinary hadrons, do not survive a more careful dynamical treatment [6].

Another binding mechanism is based on the flavor independence of the confining interaction. In a given static potential $V\left(\boldsymbol{r}_{1}, \ldots\right)$, the asymmetric mass configurations $(Q Q \bar{q} \bar{q})$ tend to be lower than the threshold $2(Q \bar{q})$ if the mass ratio is large enough [7]. This is the same favorable breaking of symmetry which makes the hydrogen molecule much more stable than the positronium molecule, in the case where the potential is taken as the Coulomb interaction (see, e.g., [8] for references).

Now, the determination of the critical mass ratio $M / m$ at which $(Q Q \bar{q} \bar{q})$ becomes stable, and the existence of other multiquark systems depend crucially on questionable assumptions on the multiquark potential. However successful is a potential $v(r)$ for the spectrum of quarkonium, its extrapolation to baryons and multiquarks remains, indeed, somewhat risky.

There are interesting attempts [9] to describe mesons and baryons simultaneously with the potential energy of the latter systems taken as

$$
V\left(\boldsymbol{r}_{1}, \boldsymbol{r}_{2}, \boldsymbol{r}_{3}\right)=\frac{1}{2}\left[v\left(r_{12}\right)+v\left(r_{23}\right)+v\left(r_{31}\right)\right],
$$

where $r_{i j}$ is the relative distance between particles $i$ and $j$. It is tempting to extrapolate this potential as

$$
V\left(\boldsymbol{r}_{1}, \ldots\right)=-\frac{3}{16} \sum_{i<j} \tilde{\lambda}_{i} \cdot \tilde{\lambda}_{j} v\left(r_{i j}\right)
$$

to higher multiquark systems, and benefit from the fewbody techniques with pairwise potentials. This was the basis of most multiquark calculations, so far. However, the success of the ansatz (1) is probably accidental, since there are many indications that if the quark-antiquark confinement is linear, $v(r)=\lambda r$, the true confining interaction for three quarks in a baryon is more likely the socalled $Y$-shape potential $[10,11]$

$$
Y\left(\boldsymbol{r}_{1}, \boldsymbol{r}_{2}, \boldsymbol{r}_{3}\right)=\lambda \min _{k}\left(r_{k 1}+r_{k 2}+r_{k 3}\right),
$$




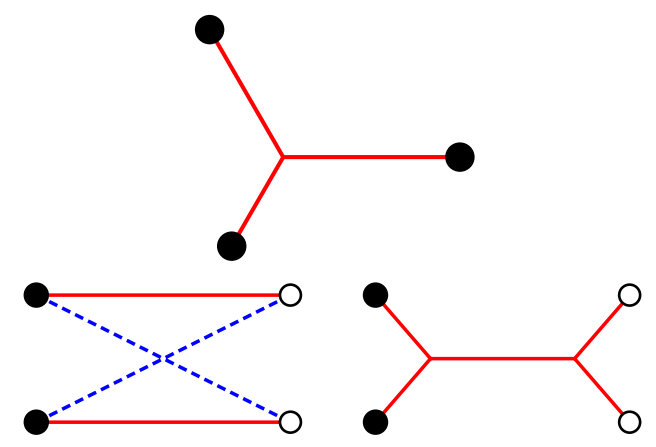

FIG. 1 (color online). String model for three-quark confinement (top), and for four quarks (bottom): flip-flop (left) and "butterfly" (right), an alternative configuration that is favored when the quarks (closed circles) are well separated from the antiquarks (open circles).

where the sum of distances from a junction $k$ to the three quarks is minimized, as in the well-known problem of Fermat and Torricelli, also reported to as the Steiner problem [12]. It is schematically represented in Fig. 1. From this point of view, the success of the empirical model (1) comes from the perimeter in a triangle being nearly equal to twice the minimal sum of distances $Y$.

The analog of (3) for four-quark systems is rather complicated. Some diagrams generalizing the $Y$-shape string have been drawn in the time of baryonium (see, e.g., [13]), but they were not followed by any thorough four-body calculation. The dynamics of systems made of two quarks and two antiquarks has been discussed by several authors, for instance [14-16], where we found guidance. The model we use for an exploratory study is restricted to the sole confinement. The short-range Coulomblike interaction, as well as the spin-dependent terms are neglected altogether. We also take the nonrelativistic limit. The interaction which is adopted here for a careful estimate of the fourbody energy is a combination of the string limit used for mesons and for baryons, and will be referred to as the "string potential." It is schematically pictured in Fig. 1 and it reads,

$$
V_{s}=\min \left(V_{f}, V_{b}\right) .
$$

$V_{f}$ stands for the so-called "flip-flop" model

$$
V_{f}=\lambda \min \left(r_{13}+r_{24}, r_{23}+r_{14}\right),
$$

where each of the quarks 1 or 2 links to either antiquarks 3 or 4 , to minimize the sum of the two terms. It is understood here that the gluon field readjusts immediately to its minimal configuration. This is a kind of ideal BornOppenheimer limit. $V_{b}$ is the butterflylike configuration, where two quarks form a color $\overline{3}$ diquark, two antiquarks a color 3 antidiquark, which are linked by a flux tube,

$$
V_{b}=\lambda \min _{k, \ell}\left(r_{1 k}+r_{2 k}+r_{k \ell}+r_{\ell 3}+r_{\ell 4}\right) .
$$

For the $Y$-shape potential of baryons, the junction achieving the minimal energy is either one of the quarks, if the triangle linking the three quarks is flat (an angle larger than $120^{\circ}$ ) or the point from which all sides are seen under $120^{\circ}$. This gives an explicit expression in terms of the interquark distances [11]. In the case of the butterfly potential, some rigorous geometrical properties remain, but one cannot avoid some numerical minimization over some of the junction coordinates, and this slows down the four-body variational computation.

Our numerical results have been obtained using correlated Gaussians, namely, if

$$
\begin{gathered}
x_{1}=r_{2}-r_{1}, \quad x_{2}=r_{4}-r_{3}, \\
x_{3}=\frac{1}{2}\left(r_{3}+r_{4}-r_{1}-r_{2}\right),
\end{gathered}
$$

are the Jacobi variables, suitably generalized in the case of unequal masses, the trial wave function is sought as

$$
\Psi\left(\boldsymbol{x}_{1}, \boldsymbol{x}_{2}, \boldsymbol{x}_{3}\right)=\exp \left[-\sum_{i \geq j=1}^{3} a_{i j} \boldsymbol{x}_{i} \times \boldsymbol{x}_{j}\right]+\cdots,
$$

where the ellipses are meant for terms deduced from the first Gaussian to restore the proper symmetry of the whole wave function. This method is widely used in nuclear physics and $a b$ initio quantum chemistry [17]. The range parameters $a_{i j}$ are optimized numerically.

For cross-check, we also use an exponential function borrowed from the famous paper in which the stability of the positronium molecule was demonstrated [18], namely

$$
\Phi=\exp \left[-a\left(r_{13}+r_{24}\right)-b\left(r_{14}+r_{23}\right)\right]+\cdots,
$$

but the calculation has been restricted to the case of the flip-flop interaction.

Note that since the potential is proportional to the distance, the virial theorem states that the kinetic, $\langle K\rangle$, and potential, $\langle V\rangle$, contributions to the energy $E=\langle K\rangle+\langle V\rangle$ are in ratio $\langle V\rangle=2\langle K\rangle$. This also holds for the variational energy if the space of trial functions is globally invariant under rescaling, see, e.g., [8]. Hence instead of minimizing $\langle K\rangle+\langle V\rangle$ with, say, $n$ parameters, it is sufficient to minimize $\left(4\langle K\rangle\langle V\rangle^{2} / 27\right)^{1 / 3}$ with $n-1$ parameters.

By scaling, the string constant can be set to $\lambda=1$ without loss of generality, and one of the masses also taken as the unit of mass, $m=1$. In these units, the ground-state energy of a meson with both quark and antiquark of mass $m=1$ is $E_{2}(1,1)=2.338$ (the opposite of the first zero of the Airy function), and for a meson of masses $m_{1}$ and $m_{2}$, it is

$$
E_{2}\left(m_{1}, m_{2}\right)=E_{2}(1,1)\left(\frac{2 m_{1} m_{2}}{m_{1}+m_{2}}\right)^{-1 / 3} .
$$

We first consider the case of equal masses, in the simple flip-flop model with exponential functions. The 
meson energy, if calculated variationally from $\phi(r)=$ $\exp (-\alpha r), \alpha$ being adjusted, is $E_{2}=2.476$. This is not very good, since the cusp in this wave function is absent from the exact wave function. With a combination of two such exponentials, the meson energy is improved to $E_{2}^{\prime}=$ 2.353. Now with the wave function (9), and the flip-flop potential, the minimal energy for the $(q q \bar{q} \bar{q})$ system is found at $E_{4}=4.872$. The observation that $E_{4}<2 E_{2}$, corresponding to stability within the simplest approximation in each sector, is an indication that the flip-flop potential tends to bind the system.

If this computation is now carried out with the Gaussian wave function (8), one obtains a variational energy $E_{4}=$ 4.644, which is below the threshold for spontaneous dissociation. The string model slightly lowers this energy, to $E_{4}=4.639$.

If the calculation is done with the trial wave function (8), but with the restriction $i=j$ which implies that only $x_{i}^{2}$ terms are allowed, the minimum of the energy is found appreciably larger, $E_{4}=4.797$ for $M / m=1$ and $E_{4}=$ 4.342 for $M / m=2$. Comparing these results with Table I, the four-quark states would be above the dissociation threshold. This approximation, which consists of neglecting the relative angles between the different Jacobi coordinates and therefore internal relative orbital angular momentum, was used in Ref. [16] with a similar "string potential," leading the authors to conclude that no bound exotic states exist (even for the sole confinement potential, see Table I of Ref. [16]).

We now introduce some symmetry breaking in the kinetic energy, and consider the configurations $(Q Q \bar{q} \bar{q})$ and $(Q \bar{Q} q \bar{q})$ with two different masses, $M$ and $m$. The results are shown in Table I. Clearly, as $M / m$ increases, a deeper binding is obtained for the flavor-exotic $(Q Q \bar{q} \bar{q})$ system. For the hidden-flavor $(Q \bar{Q} q \bar{q})$, however, the stability deteriorates, and with our variational approximation, for $M / m \gtrsim 1.2$, the system becomes unbound with respect to its lowest threshold $(Q \bar{Q})+(q \bar{q}) .{ }^{1}$ The amount of binding, independent of any scale factor, is well measured by the dimensionless coefficient defined by

$$
E_{4}=(1-u) T_{4}
$$

linking two-body and four-body energies. A plot of $u$ is given in Fig. 2, as a function of the mass ratio $M / m$.

Our main conclusions and comments are in order:

(i) With a string model including four-body forces, inspired by the strong-coupling regime of QCD, the ground-state energy of the system made of two quarks and two antiquarks of equal masses is found below the dissociation threshold.

\footnotetext{
${ }^{1}$ An energy above the threshold simply means that the system is unbound within our variational approximation, suggesting that the minimum of the Hamiltonian is at the two-meson threshold. It would be more difficult to find an approximate mass for a possible meson-meson resonance.
}

TABLE I. Four-quark variational energy $E_{4}$ of $(Q Q \bar{q} \bar{q})$ for the different confinement models described in Eq. (4), compared to its threshold, $T_{4}=2 E_{2}(1, M)$, and variational energy $E_{4}^{\prime}$ of $(Q \bar{Q} q \bar{q})$ with the flip-flop model $V_{f}$, compared to its threshold $T_{4}^{\prime}=E_{2}(M, M)+E_{2}(1,1)$, as a function of the mass ratio.

\begin{tabular}{lcccccc}
\hline \hline & & $E_{4}$ & & $T_{4}$ & $E_{4}^{\prime}$ & $T_{4}^{\prime}$ \\
$M / m$ & $V_{f}$ & $V_{b}$ & $V_{s}$ & & $V_{f}$ & \\
\hline 1 & 4.644 & 5.886 & 4.639 & 4.676 & 4.644 & 4.676 \\
2 & 4.211 & 5.300 & 4.206 & 4.248 & 4.313 & 4.194 \\
3 & 4.037 & 5.031 & 4.032 & 4.086 & 4.193 & 3.959 \\
4 & 3.941 & 4.868 & 3.936 & 3.998 & 4.117 & 3.811 \\
5 & 3.880 & 4.754 & 3.873 & 3.942 & 4.060 & 3.705 \\
\hline \hline
\end{tabular}

(ii) For the flavor-exotic $(Q Q \bar{q} \bar{q})$, binding becomes better when the mass ratio increases.

(iii) For the cryptoexotic $(Q \bar{Q} q \bar{q})$, the effect of symmetric breaking is opposite. In atomic physics, while $\left(p, p, e^{-}, e^{-}\right)$is more stable than the positronium molecule, the configuration $\left(M^{+}, M^{-}, m^{+}\right.$, $m^{-}$) becomes unstable (besides internal annihilation) for $M / m>2.2$ [8].

(iv) For $(1,2,3,4)=(Q Q \bar{q} \bar{q})$ configurations, we considered quarks (or antiquarks) of equal mass, for the ease of computation. We neglected the effect of statistics, i.e., our results directly apply to quarks (or antiquarks) having different flavor and about the same mass. Then the interquark interaction is really an effective potential with the gluon degrees of freedom integrated out, the analog of the nucleusnucleus effective interaction for diatomic mole-

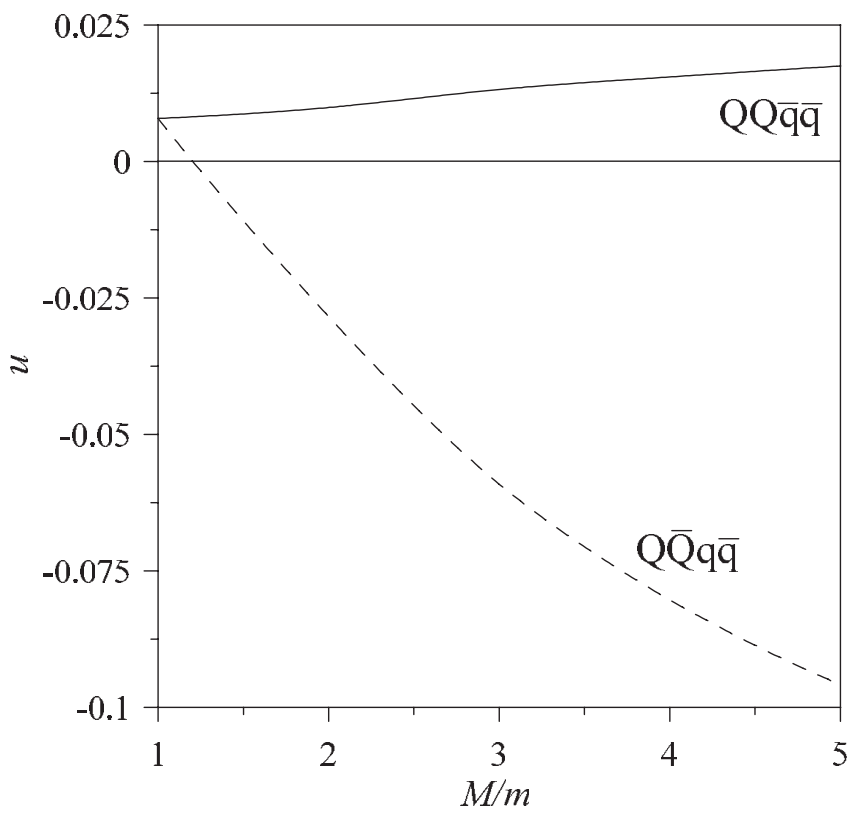

FIG. 2. Dimensionless excess of binding with respect to the threshold, $u$, as a function of the mass ratio $M / m$. 
cules in atomic physics. For genuinely identical quarks, another approach is possible, where each component of the interaction is associated to a specific color wave function, for instance $(1,2)_{3} \times$ $(3,4)_{3}$ for the "butterfly potential," $(1,3)_{1}(2,4)_{1}$ or $(1,4)_{1}(2,3)_{1}$ for each component of the "flip-flop," in an obvious notation. A formalism has been developed in Ref. [15], but it was associated to a quadratic interaction, and hence the results are not directly comparable to ours.

(v) The stability of four-quark states is demonstrated using a rather simple wave function. However, the dependence upon the angle between the Jacobi variables is crucial. Its neglect explains why stability was missed in earlier investigations.

(vi) It is delicate to compute the connected-string contribution (butterfly) to the potential, but this is not rewarding, as the dynamics of binding is dominated by the simple flip-flop term.

(vii) It would be interesting to analyze the results of lattice QCD in terms of the strength parameters associated to our string potential, and also in terms of departures from this simple ansatz. For a discussion on the multiquark interaction on lattice, see, e.g., [19].

In brief, the question of saturation raised in the early days of the quark model looks even more open today. Chromoelectric models based on simple pairwise forces (2) do not bind tetraquarks, except in the limit of high mass ratios for $(Q Q \bar{q} \bar{q})$. Our result indicates that with a more plausible scenario for the spin-independent potential, the starting point is stability.
The present study is focused on the role of confining forces alone, to demonstrate that a string model of confinement leads to results which differ qualitatively from these obtained from additive pairwise potentials. It remains to examine whether the necessary refinements will spoil or improve this binding. Among them, let us mention: relativistic effects, spin-dependent terms, Fermi statistics for identical quarks, long-range Yukawa forces between clusters (by itself this mechanism might produce binding, as shown in the "molecular" models of the X(3872) [20]). Although the many-body confinement forces discussed in this paper play a role for any four-quark system, their contribution to generate binding should be more evident for quarks of the second generation. For light quarks or antiquarks, chromomagnetic effects or their analogs in chiral dynamics are important and presumably dominate the issue of stability; if the threshold includes a pion, it is obviously difficult to imagine a four-quark state below that threshold. For very heavy quarks, the Coulomb term dominates the spin-independent interaction: the problem of stability belongs then to a well-studied class of models with additive pairwise forces [7]. It is hoped that the encouraging results obtained with the string model for confinement will stimulate intensive investigations of multiquark systems in more refined constituent quark models with phenomenological applications to hadron spectroscopy. It is our intent to participate to this enterprise.

This work has been partially funded by Ministerio de Ciencia y Tecnología under Contract No. FPA2007-65748 and by Junta de Castilla y León under Contract No. SA016A07.
[1] J. J. J. Kokkedee, The Quark Model, Frontiers in Physics (Benjamin, New York, 1969).

[2] A. T. M. Aerts, P. J. G. Mulders, and J. J. de Swart, Phys. Rev. D 17, 260 (1978).

[3] R. L. Jaffe, in The Gregory Breit Centennial Symposium, edited by V. W. Hughes et al. (World Scientific, Singapore, 1999), pp. 125-144.

[4] R. L. Jaffe, Phys. Rev. Lett. 38, 195 (1977).

[5] C. Gignoux, B. Silvestre-Brac, and J.-M. Richard, Phys. Lett. B 193, 323 (1987); H. J. Lipkin, Phys. Lett. B 195, 484 (1987).

[6] J.L. Rosner, Phys. Rev. D 33, 2043 (1986); G. Karl and P. Zenczykowski, Phys. Rev. D 36, 2079 (1987); 36, 3520 (1987); T. Sakai, K. Shimizu, and K. Yazaki, Prog. Theor. Phys. Suppl. 137, 121 (2000).

[7] J.-P. Ader, J.-M. Richard, and P. Taxil, Phys. Rev. D 25, 2370 (1982); L. Heller and J. A. Tjon, Phys. Rev. D 35, 969 (1987); J. Carlson, L. Heller, and J. A. Tjon, Phys. Rev. D 37, 744 (1988); H. J. Lipkin, Phys. Lett. B 172, 242 (1986); D. M. Brink and Fl. Stancu, Phys. Rev. D 57, 6778
(1998); B. A. Gelman and S. Nussinov, Phys. Lett. B 551, 296 (2003); J. Vijande, F. Fernandez, A. Valcarce, and B. Silvestre-Brac, Eur. Phys. J. A 19, 383 (2004); D. Janc and M. Rosina, Few-Body Syst. 35, 175 (2004).

[8] E. A. G. Armour, J.-M. Richard, and K. Varga, Phys. Rep. 413, 1 (2005).

[9] D. P. Stanley and D. Robson, Phys. Rev. Lett. 45, 235 (1980); J. M. Richard, Phys. Lett. 100B, 515 (1981); Harry J. Lipkin, Phys. Lett. B 171, 293 (1986).

[10] X. Artru, Nucl. Phys. B85, 442 (1975); Hans Gunter Dosch and V.F. Muller, Nucl. Phys. B116, 470 (1976); D. Gromes and I. O. Stamatescu, Z. Phys. C 3, 43 (1979); P. Hasenfratz, R. R. Horgan, J. Kuti, and J.-M. Richard, Phys. Lett. 94B, 401 (1980); J. Carlson, J. B. Kogut, and V. R. Pandharipande, Phys. Rev. D 27, 233 (1983); E. Bagan, J. I. Latorre, S. P. Merkuriev, and R. Tarrach, Phys. Lett. 158B, 145 (1985); M. Fabre de la Ripelle and M. Lassaut, Few-Body Syst. 23, 75 (1998).

[11] T. T. Takahashi, H. Matsufuru, Y. Nemoto, and $H$. Suganuma, Phys. Rev. Lett. 86, 18 (2001). 
[12] A. O. Ivanov and A. A. Tuzhilin, Minimal Networks (CRC Press, Boca Raton, Florida 1994).

[13] H. M. Chan et al., Phys. Lett. 76B, 634 (1978); L. Montanet, G. C. Rossi, and G. Veneziano, Phys. Rep. 63, 149 (1980).

[14] H. G. Dosch, Phys. Rev. D 28, 412 (1983); G. Martens, C. Greiner, S. Leupold, and U. Mosel, Phys. Rev. D 73, 096004 (2006).

[15] F. Lenz et al., Ann. Phys. (N.Y.) 170, 65 (1986).

[16] J. Carlson and V. R. Pandharipande, Phys. Rev. D 43, 1652
(1991).

[17] Y. Suzuki and K. Varga, Lect. Notes Phys. M54, 1 (1998).

[18] E. A. Hylleraas and A. Ore, Phys. Rev. 71, 493 (1947).

[19] A. M. Green, C. Michael, J. E. Paton, and M. E. Sainio, Int. J. Mod. Phys. E 2, 479 (1993); H. R. Fiebig, K. Rabitsch, H. Markum, and A. Mihaly, Few-Body Syst. 29, 95 (2000); F. Okiharu, H. Suganuma, and T. T. Takahashi, Phys. Rev. D 72, 014505 (2005).

[20] E. S. Swanson, Phys. Rep. 429, 243 (2006). 